Tropical Journal of Pharmaceutical Research September 2021; 20 (9): 1903-1908

ISSN: $1596-5996$ (print); 1596-9827 (electronic)

(C) Pharmacotherapy Group, Faculty of Pharmacy, University of Benin, Benin City, 300001 Nigeria.

Original Research Article

http://dx.doi.org/10.4314/tjpr.v20i9.17

\title{
Asiatic acid exerts neuroprotective effect against hypoxic- ischemic brain injury in neonatal rats via inhibition of oxidative damage
}

\author{
Ying Wang', Huiping Wang², Pu Zhao ${ }^{3}$, Jiwen Cheng1, Wei Gong', Juan \\ Zhang ${ }^{4 *}$ \\ ${ }^{1}$ Department of Pediatric Surgery, ${ }^{2}$ Department of Neonatology, the Second Affiliated Hospital of Xi'an Jiaotong University, \\ Xi'an 710004, ${ }^{3}$ Department of Neonatology, Shaanxi Provincial People's Hospital, Xi'an 710068, ${ }^{4}$ Department of Neonatology, \\ Northwest Women and Children's Hospital, Xi'an 710061, Shaanxi, China
}

*For correspondence: Email: dy3893@126.com; Tel: 0086-13435678226

Sent for review: 21 April 2020

Revised accepted: 11 August 2021

\begin{abstract}
Purpose: To investigate the effect of asiatic acid on hypoxic ischemia-induced injury in neonatal rats, and the underlying mechanism of action.

Methods: Hypoxic-ischemia (HI) neonatal rat model was established via permanent ligation of the carotid artery, followed by hypoxia (exposure to $8 \%$ oxygen and $92 \%$ nitrogen) for $24 h$. Immunofluorescence, using fluorescence microscope, was used for the determination of expressions of p-TAK1, NeuN and GFAP. Western blotting was used for assaying protein expression levels, while TUNEL assay was employed for the measurement of apoptosis.

Results: Treatment of rats with asiatic acid prior to $\mathrm{HI}$ effectively prevented up-regulation of pTAK1 and decreased the count of p-TAK1-containing astrocytes. The proportion of NeuN containing p-TAK1 in HI rat brain cortex was significantly reduced by asiatic acid $(p<0.05)$. Treatment of rats with asiatic acid suppressed $\mathrm{HI}$-induced up-regulation of pJNK expression. The HI-induced increase in the expression levels of caspase-3, $p 53$ and $p$-c-Jun in rat brain cortex were reversed by asiatic acid $(p<0.05)$. The HImediated up-regulation of expressions of $p$ - JNK, caspase-3, p53 and p-c-Jun in rat brain cortex were inhibited significantly by NG25. Asiatic acid treatment also significantly alleviated HI-mediated increase in apoptosis of neurons in rat brain cortex, when compared to model group $(p<0.05)$

Conclusion: These findings suggest that asiatic acid prevents HI-induced brain injury in neonatal rats via inhibition of neuronal apoptosis. Moreover, it inhibits TAK1 activation, suppresses $p$-JNK expression and targets pro-apoptotic factors in brain cortex. Therefore, asiatic acid may be a therapeutic agent for the management of HI-induced brain injury.
\end{abstract}

Keywords: Hypoxic-ischemic, Neuroprotection, Epilepsy, Therapeutic, Apoptosis

This is an Open Access article that uses a funding model which does not charge readers or their institutions for access and distributed under the terms of the Creative Commons Attribution License (http://creativecommons.org/licenses/by/4.0) and the Budapest Open Access Initiative (http://www.budapestopenaccessinitiative.org/read), which permit unrestricted use, distribution, and reproduction in any medium, provided the original work is properly credited.

Tropical Journal of Pharmaceutical Research is indexed by Science Citation Index (SciSearch), Scopus, International Pharmaceutical Abstract, Chemical Abstracts, Embase, Index Copernicus, EBSCO, African Index Medicus, JournalSeek, Journal Citation Reports/Science Edition, Directory of Open Access Journals (DOAJ), African Journal Online, Bioline International, Open-J-Gate and Pharmacy Abstracts

\section{INTRODUCTION}

Hypoxia-ischemia $(\mathrm{HI})$ injury has been reported in about 6 out of 1,000 births worldwide, leading to chronic disability and high mortality [1]. In humans, $\mathrm{HI}$ results in perinatal injury to brain which ultimately causes seizures, impaired learning, cerebral palsy and epilepsy [2]. It has been reported that the mechanism involved in neonatal brain injury is different from that 
associated with brain injury in adults [3]. Induction of $\mathrm{HI}$ activates apoptosis in neonatal rats, thereby causing injury and impairing brain development [4]. Therefore, brain injury associated with $\mathrm{HI}$ induction may be effectively prevented through targeting of apoptotic pathways.

Mitogen-activated protein kinase (MAPK), a member of TGF- $\beta$-activated kinase-1 (TAK1) family is activated by morphologic proteins of the bones and TGF- $\beta$ [5]. Under the influence of some stressors, the activated TAK1 plays crucial role in the up-regulation of IKB kinase and nuclear factor-KB pathways [6]. Moreover, c-Jun $\mathrm{N}$-terminal kinase (JNK) and p38 MAPK stress factor-associated pathways are activated by TAK1 [6]. It has been reported that TAK1 plays a major role in cell death regulation after activation by different stimuli [7]. In pulmonary and thyroid carcinoma cells, TAK1-JNK pathway has been found be vital in activation of apoptosis [8,9]. Studies have demonstrated that targeting of TAK1-JNK pathway may be of vital therapeutic significance for different kinds of disorders and cancers [10]. Asiatic acid (Figure 1) is a compound that belongs to triterpenoid family consisting of five rings fused together, and it is present in Centella asiatica [11]. The compound exhibits neuroprotective properties in vitro and in vivo in animal models [11]. It has been reported that asiatic acid protected B103 neuroblastoma cells from apoptosis induced by $\beta$-amyloid and $\mathrm{H}_{2} \mathrm{O}_{2}$ exposure [12]. Moreover, glutamatemediated toxicity on rat cortical cells was prevented by asiatic acid through activation of cellular antioxidant defence [13]. The present study was carried out to investigate the effect of asiatic acid on hypoxia-ischemia-induced injury in neonatal rats, as well as the underlying mechanism.

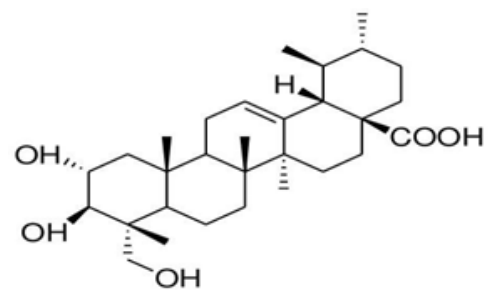

Figure 1: Chemical structure of asiatic acid

\section{EXPERIMENTAL}

\section{Establishment of rat model of $\mathrm{HI}$ neonatal}

A total of 50 neonatal rats (mean weight $=22 \mathrm{~g}$ ) were obtained from the animal house of Sichuan University, China. The neonatal rats were maintained at $23 \pm 2{ }^{\circ} \mathrm{C}$ in an environment with 12-h light/12-h dark cycle, and were given free access to feed and water. Hypoxia-ischemia (HI) was induced in the neonatal rats under diethylether anesthesia $(0.1 \mathrm{mg} / \mathrm{kg})$. After administration of the anesthesia, the rats were kept on homoisothermy bench maintained at a temperature at $37{ }^{\circ} \mathrm{C}$. The common carotid artery on right side was carefully exposed by making an incision through the midline of the neck. Then, 5-0 silk thread was used for permanent ligation of the artery, and the rats were allowed to recover from anesthesia. After $30 \mathrm{~min}$, the pups were kept in hypoxia chambers (8\% oxygen and $92 \%$ nitrogen) at a constant temperature of $37{ }^{\circ} \mathrm{C}$ for $24 \mathrm{~h}$. Pups in the sham group were given incision, and 5-0 silk was kept around carotid artery, but ligation was not performed. Asiatic acid was intragastrically administered to the pups at doses of 2, 4 and 10 $\mathrm{mg} / \mathrm{kg} 1 \mathrm{~h}$ before $\mathrm{Hl}$ induction. The rats received $\mathrm{NG} 25$ in the right cerebral hemisphere, $30 \mathrm{~min}$. before $\mathrm{HI}$ induction. The experimental methodology used in this study was in accordance with National Institute of Health guidelines [14]. The study was approved by Animal Ethics Committee of Northwest Women and Children's Hospital, Shaanxi, China (approval number $=$ NWCH/0017/076).

\section{Immunofluorescence}

At $24 \mathrm{~h}$ after $\mathrm{HI}$ induction, the rat pups were anesthetized using ketamine injection at a dose of $100 \mathrm{mg} / \mathrm{kg}$, along with xylazine $(10 \mathrm{mg} / \mathrm{kg})$ via intraperitoneal route. The pups were sacrificed by decapitation and the brain samples were excised, perfused and subsequently fixed for 48 $\mathrm{h}$ in $4 \%$ paraformaldehyde. The brains were embedded in paraffin and cut into $3-\mathrm{mm}$ thin sections using a microtome. Then, the sections were probed for protein expression by incubating them at $4{ }^{\circ} \mathrm{C}$ with primary antibodies against p-TAK1 (CAS no. 9339, dilution 1:200); NeuN (CAS no. MAB377, dilution 1:150), and GFAP (CAS no. MAB360, dilution 1:80). Thereafter, the sections were incubated with fluorescein isothiocyanate-conjugated secondary antibody (CAS no. sc-2012) at $37^{\circ} \mathrm{C}$. The cell nuclei were dyed with 4',6-diamidino2 phenylindole, and the sections were randomly imaged at five fields using a fluorescence microscope (DTX500; Nikon Corporation, Tokyo, Japan).

\section{Western blot analysis}

At $24 \mathrm{~h}$ after $\mathrm{HI}$ induction, the rat pups were anesthetized with ketamine injection $(100 \mathrm{mg} / \mathrm{kg}$ ) and xylazine $(10 \mathrm{mg} / \mathrm{kg})$ via intraperitoneal route. The brain samples were excised, and the cortex 
and hippocampus were isolated from the right hemisphere. The tissues were washed with PBS and homogenized with RIPA lysis buffer (Haimen, China) for $25 \mathrm{~min}$. The homogenate was centrifuged for $15 \mathrm{~min}$ at $10,000 \mathrm{rpm}$, and the protein content of the supernatant was measured using BCA protein kit (Beyotime Institute of Biotechnology). Then, 20- $\mathrm{gg}$ protein samples were resolved using $10 \%$ SDS-PAGE and subsequently transferred onto PVDF membranes. The membranes were blocked by incubation with $5 \%$ non-fat milk and TBS containing $0.05 \%$ Tween-20 for $1 \mathrm{~h}$. Thereafter, the membrane was incubated overnight at $4{ }^{\circ} \mathrm{C}$ with primary antibodies against p-TAK1 (cat no. 9339, 1:1,000); TAK1 (cat no. 5206, 1:800); p-JNK (cat no. 9255, 1:1,000); p-c-Jun (cat no. 3270, 1:800, all from Cell Signaling Technology, Inc.); p53 (cat no. ab26, 1:200; Abcam), and caspase-3 (cat no. C9598, 1:100). Then, the membrane was washed with PBS and incubated for $2 \mathrm{~h}$ with horseradish peroxidase-conjugated secondary antibody at room temperature. Visualization of bands was carried out with electrochemiluminescence detection kit (Merck $\mathrm{KGaA}$ ), while quantification was done with Image-Pro Plus (version 6.0; Inc, Rockville, MD, USA).

\section{TUNEL assay}

TUNEL assay was employed for determination of apoptosis in brain cortex tissues of the neonatal rats at $24 \mathrm{~h}$ after $\mathrm{HI}$ induction, using DeadEnd $^{\mathrm{TM}}$ Fluorometric system (Promega Corporation, Madison, WI, USA). Cellular apoptotic nuclei were identified using darkgreen fluorescence staining of the tissues, while a fluorescence microscope was used for quantification of apoptotic cells in five randomly selected regions of the tissues.

\section{Statistical analysis}

Data are presented as mean \pm SD of triplicate measurements. The data were analyzed using SPSS version 19.0 (SPSS, Inc., Chicago, IL, USA). Differences amongst groups were determined using One-Way Analysis of Variance (ANOVA) and Tukey's post hoc test. The differences were assumed statistically significant at $p<0.05$.

\section{RESULTS}

Asiatic acid inhibited TAK1 phosphorylation in neonatal rats with $\mathrm{HI}$

There was a significant increase in expression of $\mathrm{p}$-TAK 1 in rat brain cortex at $24 \mathrm{~h}$ after $\mathrm{HI}$, when compared with the sham group (Figure 2). However, treatment of the rats with asiatic acid prior to $\mathrm{HI}$ induction effectively and dosedependently prevented up-regulation of p-TAK1 in brain cortex, relative to model group. Treatment of rats with asiatic acid at doses of 2 and $4 \mathrm{mg} / \mathrm{kg}$ significantly suppressed Hl-induced p-TAK1expression in brain cortex. In $\mathrm{HI}$ rats, asiatic acid treatment at a dose of $10 \mathrm{mg} / \mathrm{kg}$ suppressed $\mathrm{p}$-TAK1expression, relative to the sham group.

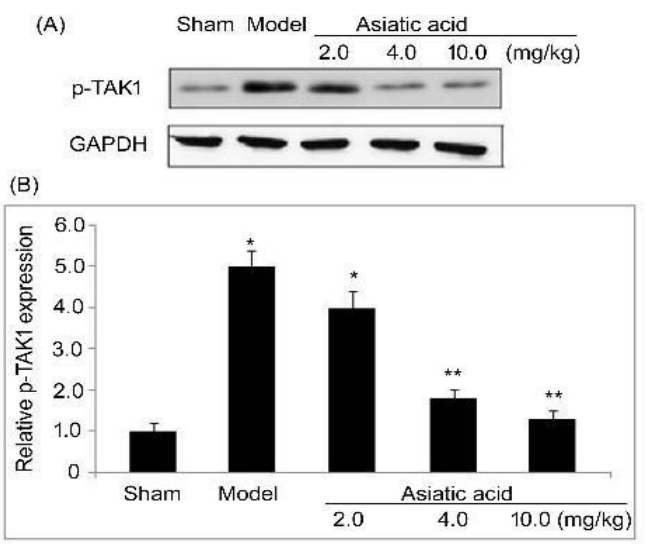

Figure 2: Effect of asiatic acid on TAK1 expression in neonatal rats. (A) TAK1 expression in brain cortex $\mathrm{HI}$ rats pre-treated with asiatic acid at doses of 2, 4 and $10 \mathrm{mg} / \mathrm{kg}$, as measured using western blotting. (B) Quantified TAK1 expression at $24 \mathrm{~h}$ after $\mathrm{HI}$ induction in brain cortex, with GAPDH as control; ${ }^{* *} p<0.018$; ${ }^{* *} p<0.09$ vs. sham

\section{Asiatic acid suppressed p-TAK1-containing} GFAP cell count in $\mathrm{HI}$ rat brain cortex

The $\mathrm{HI}$ model rats had markedly higher proportion of astrocytes containing p-TAK1 in the brain cortex than the sham group (Figure 3). The count of astrocytes containing p-TAK1 in the $\mathrm{HI}$ rat brain cortex was significantly decreased on treatment with asiatic acid at a dose of $10 \mathrm{mg} / \mathrm{kg}$.
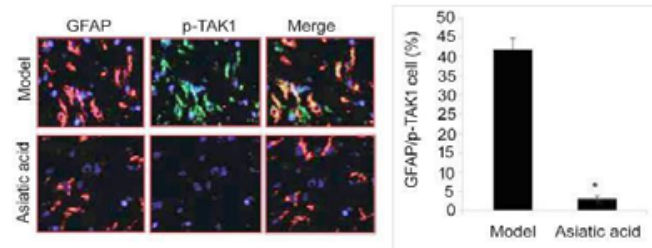

Figure 3: Effect of asiatic acid on GFAP staining in $\mathrm{HI}$ rat brain cortex. The $\mathrm{HI}$ rats were pre-treated with asiatic acid at doses of 2,4 and $10 \mathrm{mg} / \mathrm{kg}$. The tissues were subjected to double immunofluorescence GFAP (red) + p-TAK1 (green) staining. The nuclei of the cells were stained with DAPI 
Asiatic acid suppressed count of p-TAK1containing NeuN cells in HI rat brain cortex

Immunofluorescence data revealed significantly higher count of NeuN cells containing p-TAK1 in $\mathrm{HI}$ rat brain cortex than in the sham group (Figure 4). The proportion of NeuN cells containing p-TAK1 was markedly reduced in $\mathrm{HI}$ rat brain cortex on treatment with asiatic acid at a dose of $10 \mathrm{mg} / \mathrm{kg}$.
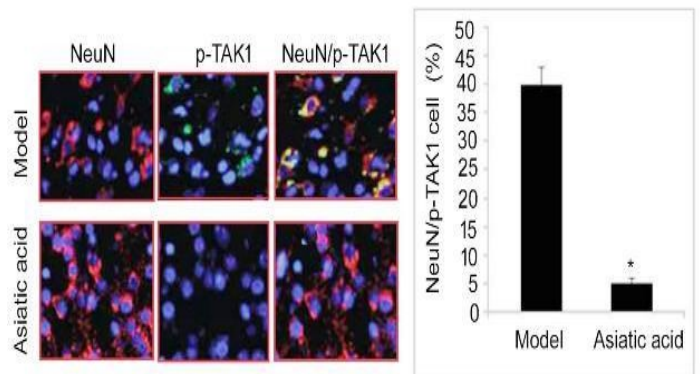

Figure 4: Asiatic acid suppressed p-JNK expression in $\mathrm{HI}$ rat brain cortex. The $\mathrm{HI}$ rats were pre-treated with asiatic acid at doses of 2, 4 and $10 \mathrm{mg} / \mathrm{kg}$. The tissue sections embedded in paraffin were subjected to double immunofluorescence NeuN (red) + p-TAK1 (green) at $24 \mathrm{~h}$ after $\mathrm{HI}$ induction

The $\mathrm{HI}$ induction significantly promoted p-JNK expression in brain cortex of the rats at $24 \mathrm{~h}$, when compared to sham group (Figure 5). However, asiatic acid treatment markedly reduced $\mathrm{HI}$-induced up-regulation of $\mathrm{p}$-JNK expression in a dose-based manner. The HIinduced increase in p-JNK expression in rat brain cortex was reversed completely on treatment with asiatic acid at a dose of 10 $\mathrm{mg} / \mathrm{kg}$. Moreover, marked elevation in levels of caspase-3, p53 and p-c-Jun were observed in the brain cortex of rats following HI. However, the $\mathrm{HI}$-induced increases in expression levels of caspase-3, p53 and p-c-Jun in rat brain cortex were reversed. by asiatic acid treatment.

\section{Effect of NG25 on expression of p-JNK in HI rat brain cortex}

Administration of NG25 (p-TAK1 inhibitor) caused a significant reduction in p-TAK1 expression in the brain cortex of $\mathrm{HI}$ rats (Figure 6 ). The HI-mediated up-regulation of $p$-JNK expression was also suppressed in rat brain cortex by administration of NG25. Moreover, the $\mathrm{HI}$-induced up-regulations in expression levels of caspase-3, p53 and p-c-Jun in rat brain cortex were markedly reversed on administration of NG25.

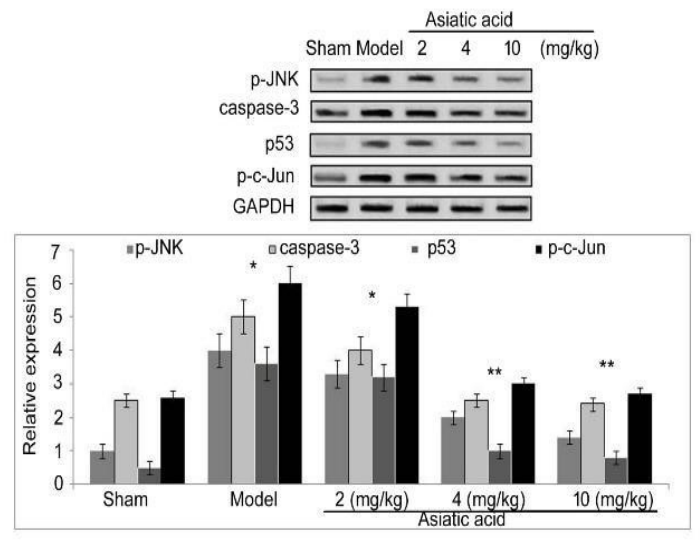

Figure 5: Effect of asiatic acid on JNK activation in $\mathrm{HI}$ rats. (A) Expression levels of p-JNK, caspase-3, p53 and $\mathrm{p}$-c-Jun in brain cortex of rats pre-treated with asiatic acid at doses of 2, 4 and $10 \mathrm{mg} / \mathrm{kg}$ prior to $\mathrm{HI}$, as measured using western blotting. (B) Quantification of protein expression levels in rat brain cortex at $24 \mathrm{~h}$ after $\mathrm{HI}$ induction, with GAPDH as control. ${ }^{* *} p<0.018 ;{ }^{* *} p<0.09$ vs. sham

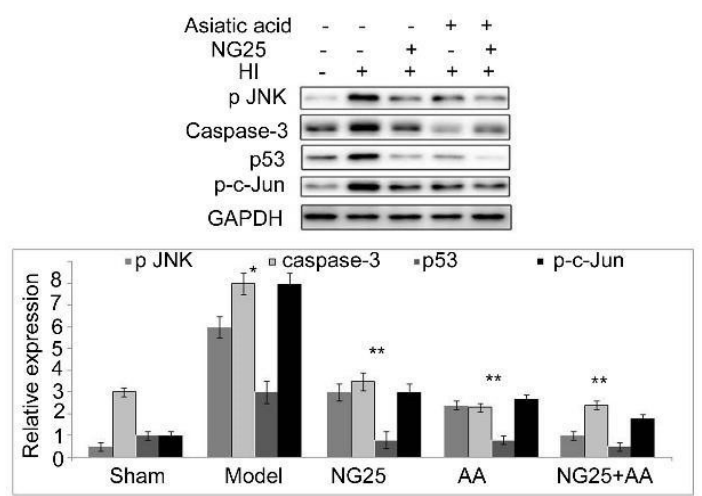

Figure 6: Effect of NG25 administration on p-JNK expression. (A) Expression levels of $p$-JNK, caspase3 , p53 and p-c-Jun in brain cortex of $\mathrm{HI}$ rats administered NG25, as measured using western blotting. (B) Quantification of protein expression levels at $24 \mathrm{~h}$ after $\mathrm{HI}$ induction in brain cortex, using GAPDH as control; ${ }^{* *} p<0.018 ;{ }^{* *} p<0.09$ vs. sham

\section{Asiatic acid prevented neuronal apoptosis induced by $\mathrm{HI}$ in rats}

TUNEL staining of brain cortex from $\mathrm{HI}$ rats showed a significant increase in neuronal apoptosis, when compared to sham group (Figure 7). However, asiatic acid treatment significantly reduced $\mathrm{HI}$-mediated increase in apoptosis of neurons in rat brain cortex in a dose-based manner, when compared to model group. Indeed, the HI-induced increase in neuronal apoptosis in rat brain cortex was completely suppressed on treatment with asiatic acid at a dose of $20 \mathrm{mg} / \mathrm{kg}$. 


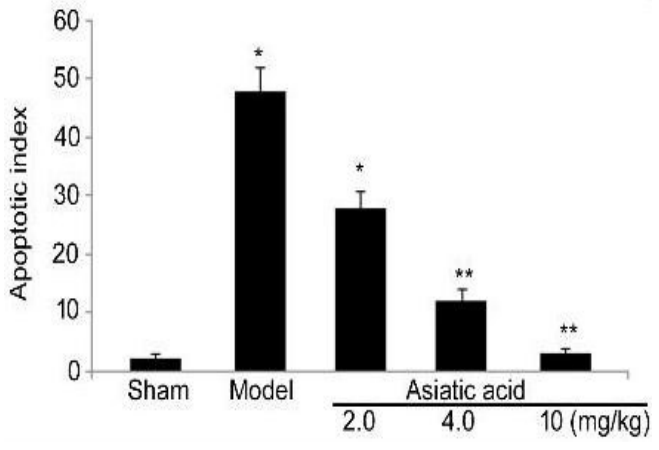

Figure 7: Effect of asiatic acid on neuronal apoptosis in rat brains. The rats were pre-treated with asiatic acid at doses of 2,4 and $10 \mathrm{mg} / \mathrm{kg}$ prior to $\mathrm{HI}$, and neuronal apoptosis in brain cortex was determined using TUNEL assay. Apoptosis index was determined by counting apoptotic cells randomly in five fields; ${ }^{* *} p$ $<0.018 ;{ }^{* *} p<0.09$ vs. sham

\section{DISCUSSION}

The TAK1-JNK pathway plays a crucial role in apoptosis induction associated with activated $T$ cells in pulmonary and thyroid cancers $[8,9]$. Following $\mathrm{HI}$ induction in developing brains, the level of p-TAK1 is increased, leading to brain injury. Therefore, the down-regulation of TAK1 is considered a potential target for treatment of cancers and many other disorders [10]. The present study found markedly higher expression of p-TAK1 in the rat brain cortex at $24 \mathrm{~h}$ after $\mathrm{HI}$ induction. However, HI-mediated enhancement of TAK1 activation was effectively suppressed in rat brain cortex by pre-treatment with asiatic acid. The $\mathrm{HI}$ induction caused a significant increase in proportion of astrocytes containing p-TAK1, and enhanced the population of NeuN containing $\mathrm{p}$-TAK1 in the rat brain cortex. However, asiatic acid treatment alleviated $\mathrm{HI}$, leading to enhancement of PTAK1-positive astrocytes and NeuN-positive cell count in the neonatal rat brain cortex.

Studies on animal models have demonstrated that neuronal apoptosis is crucial in brain injury induced by $\mathrm{HI}$ [14]. It has been reported that translocation of apoptosis-activating factors into the nucleus is related to neuronal injury in neonatal rats after hypoxia-ischemia [15]. Brain injury is inhibited by deletion of poly(ADP-ribose) polymerase-1 gene via suppression of nuclear translocation of apoptosis activation factor [16]. Apoptosis inhibition produced significant protective effect against $\mathrm{HI}$-mediated brain injury in neonatal rats $[17,18]$. In the present study, $\mathrm{HI}$ induction markedly promoted neuronal apoptosis in the brain cortex of neonatal rats. Results from TUNEL staining demonstrated that the $\mathrm{HI}$ induced neuronal apoptosis in neonatal rat brain cortex was effectively alleviated by treatment with asiatic acid. Elevated p-JNK expression was observed in the neonatal rat brain cortex following HI-induction [19,20]. However, asiatic acid markedly reversed the $\mathrm{HI}$-induced upregulation of $\mathrm{p}$-JNK expression in neonatal rats. The levels of caspase-3, p53 and p-c-Jun were enhanced in the brain cortex of rats following $\mathrm{HI}$. Treatment with asiatic acid significantly suppressed $\mathrm{HI}$-mediated up-regulation of the expressions of caspase-3, p53 and p-c-Jun in rat brain cortex. These findings indicate that asiatic acid alleviated $\mathrm{HI}$-mediated brain injury in neonatal rats via down-regulation of p-TAK1 expression. The asiatic acid-mediated prevention of $\mathrm{HI}$-induced neuronal apoptosis in neonatal rat brain was confirmed using NG25, a TAK1 inhibitor.

\section{CONCLUSION}

The findings of this study demonstrate that asiatic acid prevents $\mathrm{HI}$-induced brain injury in neonatal rats via inhibition of neuronal apoptosis. Moreover, asiatic acid inhibits TAK1 activation, suppresses p-JNK expression, and targets proapoptotic factors in brain cortex. Therefore, asiatic acid has potentials for use in the management of $\mathrm{HI}$-induced brain injury.

\section{DECLARATIONS}

\section{Acknowledgement}

This study was supported by Shaanxi Key Research and Development Plan-2018SF-246.

\section{Conflict of interest}

No conflict of interest is associated with this work

\section{Contribution of authors}

We declare that this work was done by the author(s) named in this article and all liabilities pertaining to claims relating to the content of this article will be borne by the authors. Juan Zhang conceived and designed the study; Ying Wang, Huiping Wang, Pu Zhao, Jiwen Cheng, Wei Gong collected and analyzed the data; Ying Wang, Huiping Wang, while Pu Zhao wrote the manuscript. All authors read and approved the manuscript for publication.

\section{Open Access}

This is an Open Access article that uses a funding model which does not charge readers or their institutions for access and distributed under 
the terms of the Creative Commons Attribution License (http://creativecommons.org/licenses/by/ 4.0) and the Budapest Open Access Initiative (http://www.budapestopenaccessinitiative.org/rea d), which permit unrestricted use, distribution, and reproduction in any medium, provided the original work is properly credited.

\section{REFERENCES}

1. Wachtel EV, Hendricks-Muñoz KD. Current management of the infant who presents with neonatal encephalopathy. Curr Probl Pediatr Adolesc Health Care 2011; 41: 132-153.

2. Marlow $N$, Rose AS, Rands CE, Draper ES. Neuropsychological and educational problems at school age associated with neonatal encephalopathy. Arch Dis Child Fetal Neonatal Ed 2005; 90: F380-F387.

3. Back SA, Han BH, Luo NL, Chricton CA, Xanthoudakis S, Tam J, Arvin KL, Holtzman DM. Selective vulnerability of late oligodendrocyte progenitors to hypoxia-ischemia. J Neurosci 2002; 22: 455-463.

4. Li L, Klebe D, Doycheva D, McBride DW, Krafft PR, Flores J, Zhou C, Zhang JH, Tang J. G-CSF ameliorates neuronal apoptosis through GSK-3 $\beta$ inhibition in neonatal hypoxia-ischemia in rats. Exp Neurol 2015; 263: 141-149.

5. Mihaly SR, Ninomiya-Tsuji J, Morioka S. TAK1 control of cell death. Cell Death Differ 2014; 21: 1667-1676.

6. Sato S, Sanjo H, Takeda K, Ninomiya-Tsuji J, Yamamoto M, Kawai T, Matsumoto K, Takeuchi O, Akira S. Essential function for the kinase TAK1 in innate and adaptive immune responses. Nat Immunol 2005; 6: 1087-1095.

7. Wang JS, Wu D, Huang DY, Lin WW. TAK1 inhibitioninduced RIP1-dependent apoptosis in murine macrophages relies on constitutive TNF- $\alpha$ signaling and ROS production. J Biomed Sci 2015; 22: 76.

8. Augeri DJ, Langenfeld $E$, Castle $M$, Gilleran JA, Langenfeld J. Inhibition of BMP and of TGF $\beta$ receptors downregulates expression of XIAP and TAK1 leading to lung cancer cell death. Mol Cancer 2016; 15: 27.

9. Lin P, Niu W, Peng C, Zhang Z, Niu J. The role of TAK1 expression in thyroid cancer. Int J Clin Exp Pathol 2015; 8: 14449-14456.

10. Bao D, Lu D, Liu N, Dong W, Lu YD, Qin C, Zhang LF. Tomoregulin-1 prevents cardiac hypertrophy after pressure overload in mice by inhibiting TAK1-JNK pathways. Dis Model Mech 2015; 8: 795-804.

11. Bonfill M, Mangas S, Cusidü RM, Osuna L, Piñol MT, Palazün J. Identification of tripernoid compounds of Centella asiatica by thin-layer chromatography and mass spectrometry. Biomed Chromatogr. 2006; 20 : 151-153.

12. Jew SS, Yoo CH, Lim DY, Kim H, Mook-Jung I, Jung MW, Choi H, Jung YH, Kim H, Park HG. Structureactivity relationship study of asiatic acid derivatives against beta amyloid (A beta)-induced neurotoxicity. Bioorg Med Chem Lett. 2000; 10: 119-121.

13. Lee MK, Kim SR, Sung SH, Lim D, Kim H, Choi H, Park HK, Je S, Ki YC. Asiatic acid derivatives protect cultured cortical neurons from glutamate-induced excitotoxicity. Res Commun Mol Pathol Pharmacol. 2000; 108: 75-86.

14. Haoxiang G, Can Z, Tao C, Jie Z, Shuo Y, Longfei S, Wei S, Yuhai W. Controlled Decompression Attenuates Brain Injury in a Novel Rabbit Model of Acute Intracranial Hypertension. Med Sci Monit 2019; 25:9776-9785.

15. Zhu C, Qiu L, Wang X, Hallin U, Candé C, Kroemer G, Hagberg $H$, Blomgren $K$. Involvement of apoptosisinducing factor in neuronal death after hypoxia-ischemia in the neonatal rat brain. J Neurochem 2003; 86: 306317.

16. Hagberg $H$, Wilson MA, Matsushita $H$, Zhu C, Lange $M$, Gustavsson M, Poitras MF, Dawson TM, Dawson VL, Northington F, Johnston MV. PARP-1 gene disruption in mice preferentially protects males from perinatal brain $\mathrm{J}$ Neurochem 2004; 90: 1068-1075.

17. Gu Y, Zhang Y, Bi Y, Liu J, Tan B, Gong M, Li T, Chen J. Mesenchymal stem cells suppress neuronal apoptosis and decrease IL-10 release via the TLR2/NFKB pathway in rats with hypoxic-ischemic brain damage. Mol Brain 2015; 8: 65.

18. Sun MY, Cui KJ, Yu MM, Zhang $H$, Peng $X L$, Jiang $H$. Bax inhibiting peptide reduces apoptosis in neonatal rat hypoxic-ischemic brain damage. Int J Clin Exp Pathol 2015; 8: 14701-14708.

19. Li D, Li X, Wu J, Li J, Zhang L, Xiong T, Tang J, Qu Y, Mu D. Involvement of the JNKJFOXO3a/Bim pathway in neuronal apoptosis after Hypoxic-Ischemic brain damage in neonatal rats. PLoS One 2015; 10: e0132998.

20. Nijboer $\mathrm{CH}$, Bonestroo HJ, Zijlstra J, Kavelaars A, Heijnen CJ. Mitochondrial JNK phosphorylation as a novel therapeutic target to inhibit neuroinflammation and apoptosis after neonatal ischemic brain damage. Neurobiol Dis 2013; 54: 432-444.

Trop J Pharm Res, September 2021; 20(9): 1908 